\title{
Paraplegia
}

\section{New Estimates of Traumatic SCI Prevalence: A Survey-based Approach}

\author{
C. Harvey, PhD, B. B. Rothschild, BA, A. J. Asmann, MBA, MA, T. Stripling, \\ BA \\ Rutgers Disability and Health Economics Research, Bureau of Economic Research, \\ New Fersey Hall, Room 307, New Brunswick, New fersey 08903, USA.
}

\begin{abstract}
Summary
New estimates of traumatic spinal cord injury (SCI) prevalence are developed from a survey specifically designed to identify the SCI population in both institutional and noninstitutional settings. The survey utilised a mixed-mode sampling design based on probability sampling methods. Detailed interviews were conducted with SCI persons identified in this survey. We estimate that the traumatic SCI prevalence rate in the United States is 721 cases per million, and that there are about 177000 SCI persons residing in the United States.
\end{abstract}

Key words: Spinal cord injury; Prevalence; USA.

Estimates of traumatic spinal cord injury (SCI) prevalence in the United States range from 525 cases per million population (Kurtzke ${ }^{1}$ to 1124 cases per million, based on data from the National Health Interview Survey (NHIS) in Collins. ${ }^{2}$ The most widely cited SCI prevalence rate is $906 \mathrm{SCI}$ cases per million (DeVivo et al. ${ }^{3}$ ). These estimates are derived either from a mathematical relationship among prevalence, incidence, and duration of SCI or from national population surveys which are not specifically intended to identify and estimate the SCI population.

This paper presents new estimates of the prevalence of traumatic SCI derived from a survey specifically designed to identify the SCI population in both institutional and non-institutional settings. Residential areas and institutions were sampled and queried about the presence of SCI individuals. SCI individuals identified in this effort participated in an in-depth interview covering their demographic and socioeconomic characteristics, circumstances and severity of injury, their work history (both pre- and post-injury), and their use of medical goods and services. Questions on functional limitations, activities of daily living, and lifestyle changes brought about by SCI were also included.

This approach represents a significant departure from the methodologies used 
previously to estimate the SCI population. We estimate that the SCI prevalence rate in the United States is 721 cases per million, and that there are approximately 177000 persons in the United States alive today who have sustained such an injury.

\section{SCI Survey design}

The ideal method to identify SCI persons would be to draw a random sample of individuals from the general population and then to screen for an SCI injury. However, SCI is a rare condition within the enormous population of the continental United States. These facts make the drawing of a purely random sample inefficient, expensive and infeasible.

A mixed-mode sampling design based on probability sampling methods was used to sample and survey the non-institutionalised and institutionalised populations. The design used an area probability network sample to cover the full residential SCI population and a probability list sample of long term permanent residential facilities (e.g. nursing homes) to identifiy the institutional SCI population. The design of this mixed-mode methodology is described below.

\section{Area (non-institutional) sample}

Households within pre-specified geographic areas were surveyed to develop SCI prevalence estimates for the non-institutionalised population. A total of 120 locations or primary sampling units (PSUs) representative of the continental United States as a whole were identified. Each location was selected with a known probability based on its size (number of housing units) within the continental United States, thus making projections of national SCI prevalence and related characteristics possible.

Within these locations, area segments were selected. An area segment is defined as a small, readily-identifiable cluster of housing units conforming to census boundaries. A total of 380 such segments were selected containing at least 150 housing units each. Such a sample was expected to be of a size sufficient to identify approximately 120 SCI individuals.

To minimise the costs of surveying households in the area sample, a network sampling approach was utilised. Network sampling was first pioneered in the late 1960 s and early 1970s as a way of identifying rare populations. It is premised upon selecting a household with known probabilities and asking a household informant to report for him or herself, other household members and a network of other nonhousehold members, typically close relatives or neighbours. Hence, through one contact, as many as three or four other contacts are also obtained.

Within each area segment, selected households were directly screened, while others were indirectly screened. The goal was to obtain information about the presence or absence of an SCI individual for all households within the area segment. Informants were asked to identify whether any members of their household had sustained a spinal cord injury; subsequently, questions were asked about adjacent and nearby neighbours within the sampled area segment. By strategically positioning screenings throughout the area, an entire segment was networked by screening only a portion of the total number of households within 
that segment. Once a potential SCI individual was identified, verification was obtained by going directly to the nominated household.

\section{Institutional sample}

To identify SCI persons who permanently reside in long term care institutions, we chose a probability sample of nursing and long term care health facilities from comprehensive lists of VA and non-VA facilities located in the 120 PSUs used in the area sample. The likelihood of an institution being home to an SCI person was thought to vary according to facility type and its service provision capacity. VA long term care hospitals were thought to be more likely to have SCI individuals than non-VA nursing homes with physical therapy (PT) departments which, in turn, were expected to contain more cases than non-VA nursing homes without PT departments. Therefore, facility type provided the framework for the creation of three sampling strata within the institutional sample frame.

\section{Survey implementation}

The sample design described above was implemented to provide the following estimates of SCI prevalence:

- Total non-institutionalised SCI population (estimated from the area sample).

- Total institutionalised SCI population (esimated from the institutional sample).

The number of SCI cases identified in each sample provided the basis for projections of SCI prevalence. This process is described in more detail below for each sample frame.

\section{Area (non-institutional) sample}

Residential prevalence was derived from the data for all of the 65772 housing units covered by the 380 area segments in the sample. Interviewers determined the number of occupied households in an area as of late 1988 to early 1989. They also identified households in which there were SCI persons in residence. These identifications included permanent residents who may have been temporarily absent (i.e., travelling, in a short term hospital, etc.). Each identification was confirmed, if possible, with the SCI person or his/her household members.

A total of 223 individuals were directly or indirectly identified as SCI. Of these, 121 persons were confirmed as SCI; 95 of these individuals participated in an indepth interview. Twelve persons who had at first verified that they were SCI (and were subsequently interviewed) were later re-classified as not SCI, based on their responses to specific questions in the survey and subsequent follow-up questions. When contacted directly, another 77 persons affirmed that they were not SCI. We were unable to obtain direct confirmation of an SCI condition for the remaining 13 persons.

The number of SCI persons residing within each area segment was estimated based on survey results. Adjustments were made at the segment level to correct for the possibility of false positive and false negative SCI cases among those who were not interviewed or whose condition was not confirmed. Corrections were made as well for non-response and disproportionate sampling. Each sampled household was 
assigned a weight based on its probability of selection; these selection probabilities reflected the joint probability of selecting the PSU, area segment, and household within the segment. The weighted SCI responses were summed to estimate the total non-institutionalised SCI population nationally.

\section{Institutional sample}

There are more than 21000 nursing homes and long term care hospitals in the continental United States, some of which house SCI individuals. More than 500 institutions were contacted to determine whether any SCI persons resided therein and, if so, how many. In institutions where there were SCI persons, the exact number of cases was determined when in-person interviews were attempted. The number obtained at the screening stage was reconfirmed or adjusted upward or downward as necessary, depending upon the number of revealed SCI persons. In some institutions where interviews could not be taken, the initially-reported SCI roster was used to derive the prevalence estimates.

A total of 459 institutions responded to our inquiries. Of these, 65 institutions reported at least one SCI patient in residence. A total of 396 SCI persons were identified and confirmed in this sample.

Independent estimates of prevalence were calculated for VA hospitals and for non-VA nursing homes with and without PT departments. The projection for each stratum is based on the number of institutions in that stratum estimated to contain SCI persons and the estimate of the average number of SCI patients in such institutions. ${ }^{*}$ The projected estimates for the three strata are additive and represent the total estimated prevalence of institutional SIC individuals.

\section{SCI Prevalence estimates}

Estimates of traumatic SCI prevalence among both the non-institutionalised and institutionalised populations in the United States are presented in Table I, along with prevalence rates calculated using 1988 population census data.

According to our estimates, the traumatic SCI population in the United States in 1988 was 176965 persons; this translates to a prevalence rate of 721 cases per million population. Most of these individuals reside in private households. While the institutionalised SCI population comprises a very small component of the total SCI population (i.e. about $2 \cdot 6 \%$ ), the rate of SCI prevalence among the institutionalised population is much higher than among persons residing in private households.

Before analysing these results and comparing them with previous estimates of the SCI population, we first comment on the problem of SCI case ascertainment and its impact on our prevalence estimates.

\footnotetext{
*A few nursing homes ( 2 in the VA sample and 1 in the non-VA with PT department sample) reported unusually large SCI caseloads, relative to other facilities in these strata. These outlying observations were excluded from calculating mean SCI caseload per facility; they were, however, included in the total SCI caseload from which institutionalised SCI prevalence was calculated.
} 
Table I SCI prevalence estimates total SCI population and prevalence rates 1988

\begin{tabular}{lrc}
\hline & $\begin{array}{c}\text { Number of } \\
\text { SCI persons }\end{array}$ & $\begin{array}{c}\text { Prevalence } \\
\text { per } \\
\text { million }\end{array}$ \\
\hline Non-institutionalised & 172390 & 709 \\
Institutionalised & 4575 & 1863 \\
$\quad$ VA & 754 & \\
$\quad$ Non-VA with PT dept & 861 & \\
$\quad$ Non-VA w/o PT dept & 2960 & \\
Total & $176965^{\star \star}$ & 721 \\
\hline
\end{tabular}

${ }^{*}$ Prevalence rates are calculated relative to the mid-year 1988 US resident population (US Bureau of the Census), ${ }^{4}$ divided into institutionalised and non-institutionalised components. The institutionalised resident population is estimated to be about $1 \%$ of the total resident population, based on decennial census data on the institutionalised population (US Bureau of the Census ${ }^{5}$ ).

${ }^{* *}$ Estimated with a standard error of $\pm 9 \%$ at the $95 \%$ confidence level.

\section{Problems of case ascertainment}

The study methodology, with its reliance on both direct and indirect contacts with sampled households, does not guarantee that all SCI individuals within an area segment were correctly identified. ${ }^{\dagger}$ Some sampled informants may not have been aware of the presence of an SCI individual in their neighbourhood, perhaps because the injury was relatively minor and thus not obvious. A very incapacitated SCI individual may be completely housebound; it is possible that an adjacent household, when queried about SCI in the neighbourhood, may not have known about this particular individual. To minimise the possibility of missing such SCI cases, interviewers attempted to screen at least one third of all households in an area segment directly. All suspected SCI households identified by contacts with neighbouring households were visited.

The survey relied on respondent self-reporting for positive SCI identification. That is, individuals (or, in the case of institutions, the administrative contacts) were queried directly as to whether they (or any of their residents) had sustained a spinal cord injury resulting from a traumatic event. Positive responses to the questions were taken as verification that the respondent or institutional resident qualified as spinal cord injured. No medical personnel or medical records were consulted to verify the response. Errors of two types can occur with self-reported data, false positive identification (where the respondent thinks that he/she has SCI, but does not) or a false negative identification (where the respondent doesn't know his/her diagnosis, or does not want to admit it). The presence of these two types of errors will impart an unknown bias to the SCI prevalence estimate.

For those SCI persons who were interviewed, information from the survey questionnaires was used to corroborate the self-reported SCI condition. As these questionnaires were processed, examination of responses to other questions led us to doubt that a handful of the interviewed respondents were, in fact, traumatic

†Pre-tests of the network sampling method resulted in the successful identification of 5 out of 6 known SCI cases. 
SCI. Particular attention was focused on several respondents who reported no overnight hospitalisation immediately following the injury. Some of these respondents were, in fact, never hospitalised either in the 2 years following their injury or in the last year prior to the interview date. Most of these individuals had incurred lower-level spinal injuries, (i.e., in the lumbar or sacral regions). Some reported that they had complete sensation and motor function below the point of injury. Also, some of these respondents did not report any periods of disability or interruption in work history following their injury. Responses to survey questions dealing with use of medical supplies, adaptive equipment, functional limitations and activities of daily living indicated either that the respondent was not impaired by his/her injury, or were consistent with back or spine impairments other than traumatic SCI (e.g., herniated disc).

Callbacks to these respondents yielded the following results:

- Some respondents provided additional information regarding hospitalisation periods subsequent to their injuries. These respondents were then included in our prevalence estimate.

- Some respondents were able to offer a satisfactory explanation for lack of hospitalisation immediately following injury. We also chose to include these responses in our prevalence estimate.

- Some respondents acknowledged that they were not, in fact, SCI. These responses were discounted in our prevalence estimates and treated as non-SCI cases.

- Some respondents maintained that their previous responses were, in fact, correct and that they were SCI patients.

The last group of respondents presented a particular problem. In all, eight respondents from the area (non-institutional) sample fell into this last category. While it is possible that at least some of these respondents did incur a very mild injury and may, in fact, have completely (or almost completely) recovered, we chose the conservative approach of eliminating these individuals from further consideration. Thus, the prevalence estimates presented in Table I are conservative in that they may exclude some genuine SCI cases where the injury was very slight and where the respondent may have experienced a complete or near-complete recovery. Moreover, they may also exclude some SCI persons with normal functioning who were not nominated in the network sampling process or who, when asked to verify their condition, did not choose to admit to an SCI condition.

\section{Comparisons with previous estimates}

Table II compares our PSA estimates with SCI prevalence rates and 1988 SCI population estimates based on these rates for four previous efforts to estimate SCI prevalence in the United States.

The results of our survey indicate that SCI is indeed a rare phenomenon. Across the studies listed in Table II, we tend to fall at the more conservative end. However, our SCI prevalence estimates are also conservative in the following respects:

- They do not include some possible cases of SCI which we eliminated as suspect, due to the type of responses to key questions in the interviews. Inclusion of the 
Table II Comparison of traumatic SCI prevalence estimates

\begin{tabular}{lcc}
\hline Source & $\begin{array}{c}\text { Prevalence rate } \\
\text { (per million) }\end{array}$ & $\begin{array}{c}\text { SCI population } \\
(1988)\end{array}$ \\
\hline Kurtzke $^{1}$ & 525 SCI cases & 128941 \\
PSA & 721 SCI cases & 176965 \\
DeVivo et al. ${ }^{3}$ & 906 SCI cases & 222515 \\
Ergas $^{6}$ & 1006 SCI cases & 247076 \\
NHIS $\left(\right.$ Collins $\left.^{2}\right)$ & 1124 SCI cases & 276057 \\
\hline
\end{tabular}

estimates would raise our non-institutionalised SCI count by 16207 (i.e. to 188597 persons).

- In developing institutional prevalence estimates, some institutions with very high SCI patient loads (relative to other sampled institutions) were treated as outlying observations. Had these observations been treated in the same manner as all other observations, the institutionalised SCI population estimates would increase by as much as 1670 (i.e., to 6245 persons).

- The prevalence estimates reflect prevalence in the 48 contiguous states within the United States. Residential areas and institutions in Alaska and Hawaii were not considered in the sample design. Excluding these areas should have minimal impact on our estimate.

Modification of these conservative assumptions would produce an SCI population estimate of 194842 . This 'upper-bound' estimate still falls short of 1988 SCI population estimates based on SCI prevalence rates derived from most previous studies.

While our estimates are decidedly conservative in nature, they are derived using a methodology which we believe has some distinct advantages over other estimates. Prior estimates based on mathematical relationships (Kurtzke ${ }^{1}$; DeVivo et al. ${ }^{3}$ are highly dependent on assumed or calculated incidence and mortality rates. Estimates developed from other population surveys $\left(\operatorname{Ergas}^{6}\right.$ or NHIS data found in Collins,${ }^{2}$ are not specifically designed to identify SCI persons and they may, in fact, experience case ascertainment problems of their own.

\section{Concluding remarks}

Deriving estimates of the traumatic SCI population is but the first step in a major effort to calculate the economic impacts of traumatic SCI in the United States. Estimates of the direct and indirect costs of SCI will be based on the information provided by SCI survey respondents. Complete survey questionnaires are available from 95 persons from the area (non-institutionalised) sample, while 88 surveys were completed by SCI persons contacted through the institutional sample. Another 575 SCI individuals who were identified via membership lists from specific organisations representing the interests of the disabled population also agreed to be interviewed. A complex weighting scheme assigns weights to a total of 758 respondents; the weights sum to the estimated SCI population total of 176965. The result is a statistically valid sample of the SCI population which is representative of the composition and characteristics of that population. This rich source of information will be analysed intensively to develop a comprehensive picture of the causes and consequences of SCI in the United States. 


\section{Acknowledgements}

This work was funded by the Paralysis Society of America of the Paralyzed Veterans of America (PSA). Dr G. Leon supervised the survey effort. Dr L. Wilson and Professor Monroe Berkowitz provided overall leadership and direction to the project. Research assistance was provided by Mrs Nahla El-Hawli. The authors gratefully acknowledge helpful comments from two reviewers.

\section{References}

KURTZKE JF 1975 Epidemiology of Spinal Cord Injury. Experimental Neurology 48:163-236.

Collins JG Types of Injuries and Impairments Due to Injuries. In: Vital and Health Statistics. Series 10, No. 159. U.S. Department of Health and Human Services Publication (PHS): 87-1587. Hyattsville, MD 1987: National Center for Health Statistics: pp. $48-49$.

DeVivo MJ, Fine PR, MAeTz HM, STOVER SL 1980 Prevalence of spinal cord injury: A re-estimation employing life table techniques. Archiv Neurol 37:707-708.

U.S. Bureau of the Census. Current Population Reports, Series P-23, No. 159. Population Profile of the United States: 1989. Washington, DC.: U.S. Government Printing Office. 1989: pp. 44-45.

U.S. Bureau of the Census. Statistical Abstract of the United States: 1988. 108th edition. Washington, D.C.: U.S. Government Printing Office. 1987: p. 52.

ERGAS Z 1985 Spinal cord injury in the United States: A statistical update. Central Nervous System Trauma 1985: 2:19-30. 